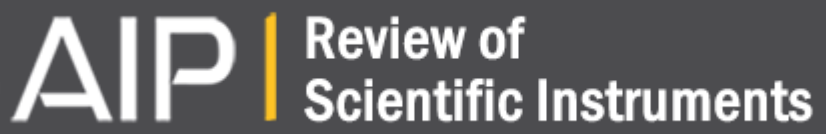

The Thirty Kilowatt Continuous Input XRay Equipment and High Constant Voltage Generating Plant of the Watters Memorial Research Laboratory at the California Institute of Technology

Jesse W. M. DuMond and J. Paul Youtz

Citation: Review of Scientific Instruments 8, 291 (1937); doi: 10.1063/1.1752317

View online: http://dx.doi.org/10.1063/1.1752317

View Table of Contents: http://scitation.aip.org/content/aip/journal/rsi/8/8?ver=pdfcov

Published by the AIP Publishing

\section{Articles you may be interested in}

The polarization technology at the High Energy Laboratory of the Joint Institute for Nuclear Research AIP Conf. Proc. 421, 411 (1998); 10.1063/1.55001

X-Ray Equipment for the Atomic Physics Laboratory

Am. J. Phys. 24, 362 (1956); 10.1119/1.1934229

Thirty Years of X-Ray Research at the General Electric Research Laboratory Am. J. Phys. 14, 71 (1946); 10.1119/1.1990804

The Cryogenic Laboratory of the California Institute of Technology. Part II Rev. Sci. Instrum. 7, 307 (1936); 10.1063/1.1752159

The Cryogenic Laboratory of the California Institute of Technology. Part I Rev. Sci. Instrum. 6, 217 (1935); 10.1063/1.1751987

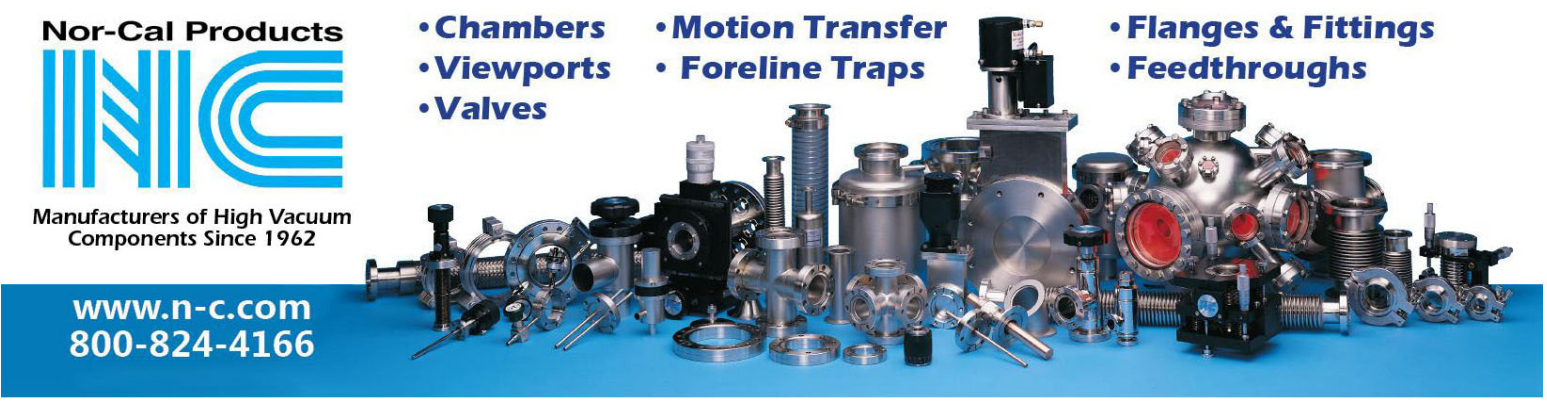




\title{
The Thirty Kilowatt Continuous Input X-Ray Equipment and High Constant Voltage Generating Plant of the Watters Memorial Research Laboratory at the California Institute of Technology
}

\author{
Jesse W. M. DuMond and J. Paul Youtz \\ California Institute of Technology, Pasadena, California
}

(Received May 5, 1937)

\begin{abstract}
This report describes in detail with photographs and cross-sectional views an $\mathrm{x}$-ray tube with a gyrating water-cooled target which the authors have operated continuously for periods of hours at 287,000 volts and 105 milliamperes. The theoretical predictions of DuMond, Watson and Hicks as to the ratio of safe load in moving and fixed targets seems to be roughly fulfilled by these results. The computed power output in the form of $x$-rays alone is some $3 / 4$ kilowatt. The report further describes in detail the power supply equipment consisting of a synchronous motor driven $40 \mathrm{kw}$ motor generator set giving 150 cycle current both half-waves of which, after transformation to high voltage, are rectified by two thermionic valves (filament emission 2 amperes maximum) of our own design and construction here fully described. The powerful electrical filter system to reduce the ripple to a low value and some technical problems arising from the large power rating of the equipment as well as the facilities now almost completed for stabilizing and measuring precisely the high voltage supplied to the $\mathrm{x}$-ray tube are discussed. At the outset a brief indication of the program of research work projected for this set is given.
\end{abstract}

\section{INTRODUCTION}

\section{General objectives}

$\mathrm{T}$ $\mathrm{HE} x$-ray generating equipment we are about to describe was designed primarily for the production of $\mathrm{x}$-rays of high intensity at moderate voltages up to 300 kilovolts. The possible applications we had in mind calling for such high intensities are:

1. Studies in $\mathrm{x}$-ray scattering involving spectral resolution of the radiation after scattering. This work is especially interesting as pure research since the structures of initially sharp $\mathrm{x}$-ray lines modified by scattering have been shown by DuMond and Kirkpatrick ${ }^{1}$ to give a valuable key to the statistics of electron motion in gas atoms and in solid bodies.

Precise Compton shift determinations are also projected. Ross and Kirkpatrick have shown ${ }^{2}$ that certain corrections must be applied to the Compton shift when the scatterer is other than a free electron in order to give an accurate determination of the fundamental Compton wavelength $h / m c$. The desirability of strongly exciting primary line radiations of the shortest possible wave-length is emphasized because the above

${ }^{1}$ J. W. M. DuMond, Rev. Mod. Phys. 5, 1 (1933). This article is a summary giving references to an extensive literature in the field.

${ }^{2}$ Ross and Kirkpatrick, Phys. Rev. 46, 668 (1934); 45, 223 (1934). correction can be empirically determined since it diminishes as the square of the primary wavelength and also because the above mentioned breadth of the shifted line diminishes relative to the shift with diminishing primary wave-length thus improving the precision of the shift determination as the wave-length diminishes. All such studies require very high primary $\mathrm{x}$-ray intensities.

2. Studies of the short wave-length limit of the continuous $x$-ray spectrum. These include first a precise study of the law $e V=h \nu_{\max }$ over a wide range of $V$ and $\nu$ in an effort to detect any possible minute departure from linearity and second a careful study with high spectral resolving power of the foot of the continuous spectrum very close to the limit. The high resolving power and the faintness of the radiation near the limit call for a powerful x-ray source for both of these problems. Furthermore the most complete freedom from organic contamination on the target is essential. Finally a very steady source of d.c. potential with extremely small superposed ripple and a precision means for measuring the voltage are required. The interest here arises from the existence, now quite generally recognized, ${ }^{3}$ of a discrepancy in

\footnotetext{
${ }^{3}$ Birge, Nature 137, 187 (1936). In this letter Birge points out that the discrepancy may be ascribed either (1) to the inappropriateness of the perfect crystal assumption in computing $e$ by the $\mathrm{x}$-ray crystal grating method
} 
the results of different ways of determining $e$ and $h$.

3. Absolute measurements of x-ray energy and momentum. The present equipment should make possible a very precise determination of the absolute energy of a given beam of $x$-rays by its thermal effects. With a total disposable output of about $\frac{3}{4} \mathrm{kw}$ in $x$-rays alone the thermal effects on the lead absorbers used to shield the tube are very appreciable. We also hope to try some measurements on the mechanical pressure exerted on an absorbing screen by an x-ray beam and preliminary calculations show that this should be possible with the present available power.

4. Technological applications such as radiography of heavy metal parts, Debye-Scherrer diffraction diagrams of materials, $x$-ray spectroscopic applications to chemical analysis, $x$-ray absorption measurements and many other $x$-ray problems find their chief limitation in the present existing available intensities and these should in many cases furnish interesting uses for the present equipment.

\section{The X-Ray Tube}

\section{Theories of mobile focal spots}

In the tube here described the great and continuous concentration of power on the small focal spot (of about 0.5 to $1 \mathrm{~cm}$ in diameter) would melt the surface of the target in spite of the copious supply of cooling water applied to the back surface (thickness front to back about $2.5 \mathrm{~mm}$ ) were it not for the motion imposed on the target so that the impinging stream of electrons sweeps over a circular path. The area of this path is from ten to fifteen times the area of the focal spot itself.

Several tubes in which rotating targets have been used to permit an increased power input have already been built by various designers. ${ }^{4}$

or (2) that the theoretical formula for the Rydberg constant may be seriously in error (by a factor of order $1+\alpha$ ). DuMond and Bollman have since shown by a crystal powder method which avoids the first pitfall above mentioned that this pitfall cannot vitiate the values of $e$ obtained with macroscopic crystals by a sufficient amount to explain the discrepancy, Phys. Rev. 50, 524 (1936).

${ }^{4} \mathrm{~A}$. Bouwers, Physica 10, 125 (1930). Bouwers's tube is designed for use in snapshot radiography advantage being taken of the heat capacity of a massive anode to store the energy of each exposure which is subsequently dissipated slowly by a radiative means, Such a tube does not permit
None of these to our knowledge has been designed to permit continuous power inputs approaching those of the present equipment. Alex Müller gives theoretical results ${ }^{5}$ for the limiting power input which will just fail to melt a target where the focal spot is either moving or fixed with respect to the target surface and where the power is applied either steadily or impulsively (as in snapshot radiography). Müller's treatment of the thermal theory for moving focal spots assumes an infinite target of given heat conductivity with a region remote from the focal spot held at fixed temperature (that of the refrigerating agency). The fact that in real targets the motion of focal spot relative to the target surface must occur in a closed path returning periodically to deliver heat to the same point is consciously neglected by this author. Müller's theory on the other hand takes very elaborate account of the spatial distribution of heat flow according to the differential equation of thermal conduction.

DuMond, Watson and Hicks on the contrary have developed a theory ${ }^{6}$ which takes account of the periodic reheating of every point in the path of the focal spot so that a steady state of thermal oscillation is established. To the senior author of these papers the spatial distribution of heat flow seems in a real target a relatively unimportant detail since obviously the correct design calls for the shortest possible length of thermal path in the metal from the focal spot to the cooling water, a path whose length in our present design is short compared to the linear dimensions of the spot, and hence the heat can, with little error, be assumed to flow in parallel lines normal to the target surface and to the water cooled inner surface. On the contrary the finite length and area of the cyclic path swept out by the focal spot seems a most essential consideration, for it is clear that even with infinite speed the ratio of safe power input for moving and fixed targets cannot exceed the ratio of areas covered. Thus a theory neglecting

of high continuous power input but is perfectly adapted for its special purpose. Clay, Proc. Phys. Soc. 46, 703 (1934) has designed a five-kilowatt rotating anode tube that is water cooled. See also reference 7 .

${ }_{5}$ Alex Müiler, Proc. Roy. Soc. 117, 30 (1927); 125, 507 (1929); 132, 646 (1931).

${ }^{6}$ DuMond, Watson and Hicks, Rev. Sci. Inst. 6, 183 (1935). 


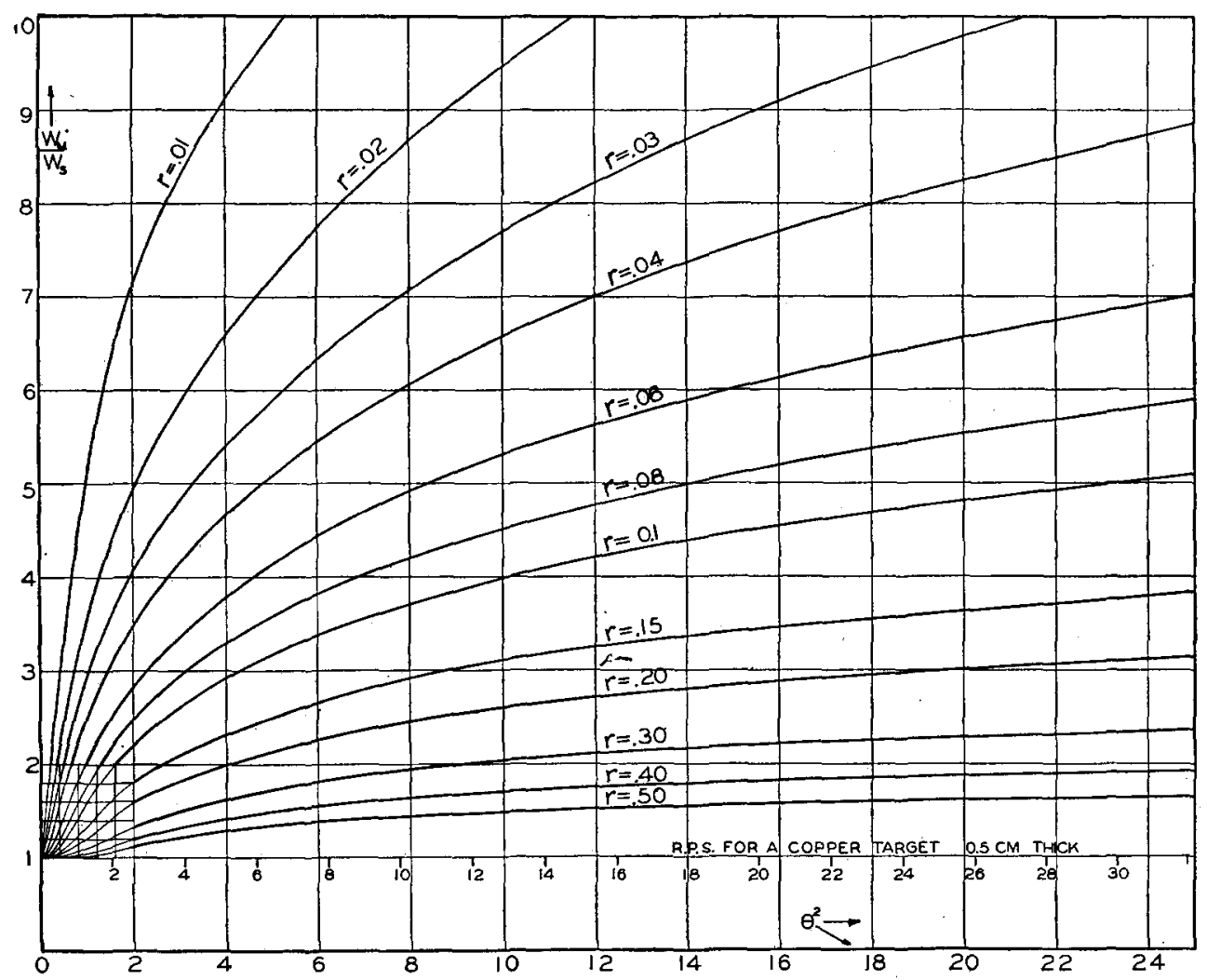

FIG. 1. Safe power input ratio for moving and fixed focal spots as a function of $r$ (whose definition is explained in Fig. 2) and of a parameter $\theta^{2}$ which is proportional to the frequency of the cyclic motion of the focal spot and depends on certain target constants and dimensions. $\theta^{2}=\left(\pi d^{2} \rho \sigma / c\right) \times$ cycles per sec. $d$ is the thickness of the target from front face to cooling water, $\sigma$ is the specific heat of the target material per unit mass, $\rho$ is the mass density of the target material and $c$ is its thermal conductivity. A scale reading directly in cycles per second has been added for the special case of a target $0.5 \mathrm{~cm}$ thick.

this point would predict far too high a safe power at high speeds. This defect in Müller's theory has been pointed out by Fournier, Gondet and Mathieu ${ }^{7}$ who have constructed a tube with a rotating disk target provided with a vacuum tight cone joint having the very slow speed of rotation of 1 rev. per sec. These authors report $10 \mathrm{kw}$ steady continued input without damage to their target.

The main results of the paper by DuMond, Watson and Hicks are shown here in the curves of Fig. 1 which give the safe power ratio for moving and fixed targets as a function of two variables, first the ratio of areas covered $(r=$ fixed area/total area swept out in motion) and second a parameter, $\theta$, proportional to the cycles

\footnotetext{
${ }^{7}$ Fournier, Gondet and Mathieu, Communication at Paris meeting of the French Physical Society, Dec. 18 (1936) see J. de phys. 8, 171S (1937).
}

executed on the target per second and involving the thermal characteristics of the target material. Fig. 2 shows the geometry of the focal spot (assumed to be a sharply bounded circle with uniform heat distribution) and of the path which it sweeps out. The exact definition of $r$ is $r=\tau_{1} / \tau_{2}$ where $\tau_{1}$ and $\tau_{2}$ are intervals of time proportional to the angles so marked in Fig. 2.

In our tube the target face does not rotate but performs approximately a circular motion of translation. This device obviates the necessity for rotating ground vacuum joints or packing glands but requires circular symmetry in the focal spot. A rotating target on the other hand permits the use of a rectangular focal spot with its long dimension radially disposed with respect to the axis of rotation but requires a vacuum tight rotating gland. The use of a circular spot is a little less effective in radiography but it 


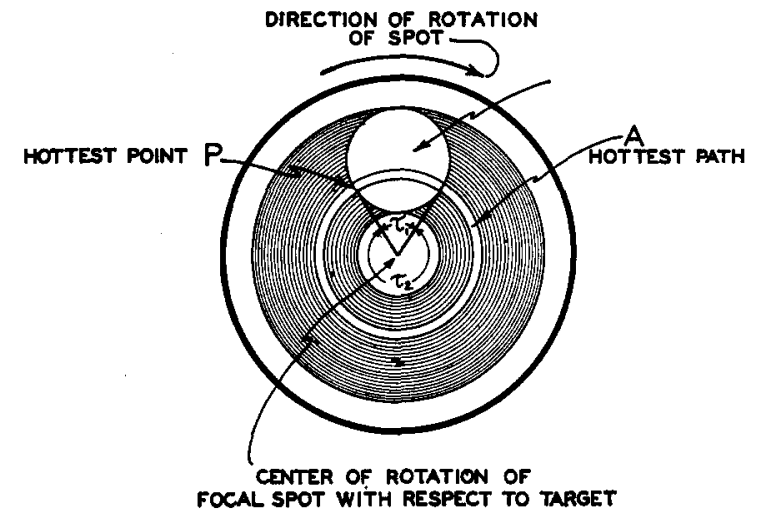

FIG. 2. The focal spot, moving relative to the face of the target sweeps out the shaded circle the hottest path being the one shown for which a given element of target surface remains longest under the bombardment. The parameter $r$ appearing in Fig. 1 is the ratio of angles $r=\tau_{1} / \tau_{2}$ (or of the intervals of time to which these angles are proportional). A disk-shaped focal spot with uniform power input per unit area out to a sharp circular boundary is assumed.

remains to be seen whether this disadvantage will out-weigh the advantage gained by avoiding the vacuum tight rotating gland. The present tube was designed for research work, already mentioned, where a focal spot of extremely small projected dimensions is no great advantage.

\section{Tube design}

Figure 3 shows in longitudinal cross section the tube itself as well as details of its more essential parts. Space limitations in the laboratory made it desirable to use a horizontal tube with ends maintained above and below ground potential by equal voltages. The over-all length of the tube as shown in this drawing from counter weight on the anticathode end to extreme cathode end is 2.6 meters.

\section{The gyrating target}

The target stem enters the vacuum through a flexible "sylphon" or metal bellows (shown at the upper left in detail). It is supported on a universal gimble support which prevents it from rotating but permits its axis to sweep out a right circular conical surface the half angle at the apex of the cone being about 1.5 degrees. We have not shown the inclination of the target stem to the axis of the tube in Fig. 3. The plane of this angle of inclination is horizontal in the position here shown both in the main cross section and in the details. The gimble joint is designed to resist the atmospheric pressure which would otherwise

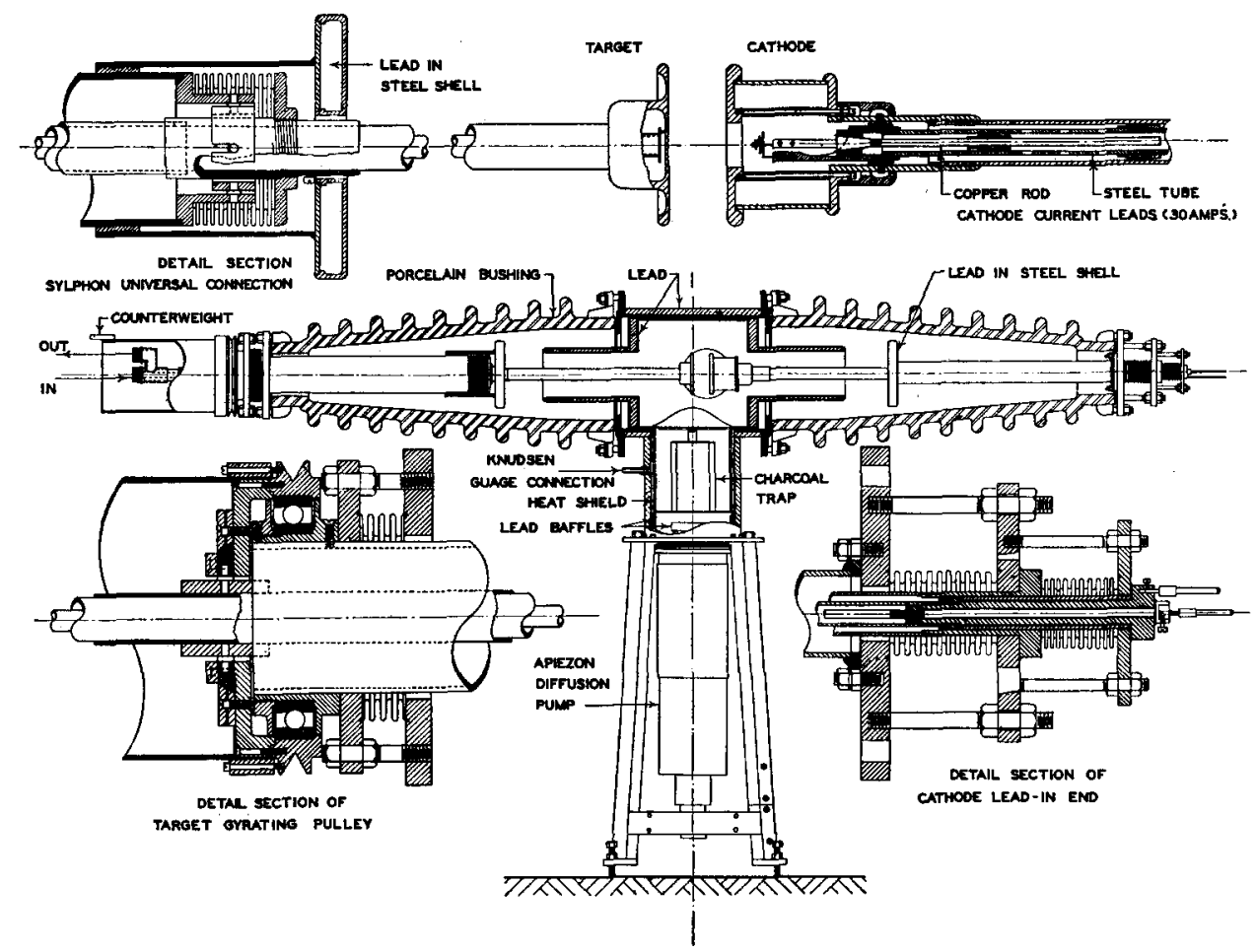

FIG. 3. Cross section of $30 \mathrm{kw}$ gyrating target $\mathrm{x}$-ray tube with some details of its construction. 
stretch the metal bellows axiàlly. As the axis of the target sweeps out this conical motion (which for brevity, lacking a better word, we shall here call gyration) the target face approximately describes a motion of circular translation. The surface of the target face is, in fact, a spherical cap with center in the apex of the above mentioned cone though this detail is of no great import. The "gyrating" motion of the target stem is given to it by means of a belt driven ball bearing pulley wheel mounted on the outer end of the tube and shown in detail at the lower left in Fig. 3. A carriage on the outward face of this wheel carries in trunions a bronze journal bearing sleeve which can be set eccentric to the axis of rotation of the wheel by sliding the carriage to the desired position and clamping it. This journal sleeve carries the target stem around in the gyrating motion. It does not, however, bear directly on the target stem itself but on another steel sleeve, a permanent part of the assembly, which carries the target stem inside it and from which the target and its stem can be removed and replaced by another target and stem if desired. This affords ready interchange of targets of different materials, each target assembly proper consisting only of the 1 -inch shelby steel tube silver soldered to the target head of copper or other material provided with the appropriate material on its front surface on which the radiation is to be excited. Our present target which has withstood 30 kilowatts without damage is of gold, coated on a copper base by an amalgamation process with mercury. ${ }^{8}$ The target

${ }^{8} \mathrm{~A}$ few cc of distilled mercury are warmed gently in a test tube under a ventilating hood with a sufficient quantity of pure gold (sheet or filings) to furnish the desired size and thickness of coating. When the gold has dissolved completely the pasty mass of amalgam is squeezed in a chamois bag to express all the free mercury, leaving a lump resembling butter in consistency. The copper target surface to be gilded is thoroughly cleaned mechanically in the lathe with clean grease-free emery cloth. (Attempts to clean it chemically and to preamalgamate the surface, frequently recommended in books, have proven less successful in practice, we find.) The amalgam is buttered onto the copper surface immediately after cleaning with a clean springy steel spatula. It will probably not adhere at first at all. The target is now to be gently warmed (under the ventilating hood) by conduction of heat from a flame which does not play on the surface to be coated. As the target temperature rises the amalgam must be continually buttered and spread about with the knife and soon with continued heating adherence will take place. Some skill is required when this point is reached to spread the analgam uniformly before it stiffens from vaporization of the mercury too much to spread. Finally when an adherent is made vacuum tight in the permanent gyrating steel sleeve with soft solder at the outer end. The cold incoming water is carried into the target down a central axial steel tube which is heat insulated from the returning hot water in the annular space outside with a wrapping of cotton tape baked onto the outside of the steel tube with varnish. The flange on the periphery of the target which about doubles the diameter of the disk presented toward the cathode has been found necessary for two reasons. First it discourages the secondary electrons emitted or reflected from the focal spot from turning round the corner of the target and being propelled down the target stem. Before this flange was installed $\mathrm{x}$-rays generated by such electrons impinging on the lead filled steel disk $x$-ray shield in front of the sylphon universal joint were an annoying secondary source difficult to shield and the energy developed (perhaps a kilowatt) was sufficient to melt the lead in this shield which then leaked out into the tube. The second function of the flange in conjunction with the rather large diameter flat faced cathode shielding box is to prevent the gyrating motion of the target from imposing much of its effect on the direction of the field in the region of the electron beam so as to prevent the focal spot from wabbling. With the design shown no motion whatever of the pin-hole image of the focal spot can be detected on a fluorescent screen.

\section{Dynamic balancing of the gyrating target as- sembly}

To avoid objectionable vibration being transmitted from the gyrating parts into the tube as a whole we have provided a counterweight (visible at the extreme left in Fig. 3) whose static moment round the axis of the tube just neutralizes the static unbalance of the gyrating system and whose position longitudinally along the axis of the tube is chosen so as just to neutralize

uniform coating is obtained the target is warmed vigorously until every trace of mercury is expelled and the surface has a matte golden color. If the target is hollow (for water cooling) we now cast it full of molten lead or type metal alloy so that when cool the gilded face can be hammered without deformation. This hammering is indispensable, for the gilded coat produced as we describe is very spongy. Hammering however consolidates it into a uniform homogeneous mass which is very rugged indeed against both mechanical and thermal abuse. Finally the lead or type metal is melted out of the interior. 
the dynamic unbalance caused by the gyration. The neutralizing forces from the counter weight must of course be transmitted through the ball bearing on the pulley. This bearing has proven itself quite equal to the task without sign of wear. Thus very little vibration is actually transmitted into the porcelain insulating sleeves and the rest of the tube structure.

\section{Centering of target assembly}

A sylphon bellows near the extreme left-hand end of the tube in Fig. 3 furnishes flexibility so that by adjusting the nuts on the studs which surround the bellows (see detail in lower left) the entire gyrating target assembly can be orientated in its support so as to center it in the $\mathrm{x}$-ray tube.

\section{The cathode}

The cathode was redesigned many times before satisfactory operation was obtained. Our experience has shown the advantage of short thick low voltage high current cathode filaments both from the point of view of mechanical strength against deformation and on account of the increased freedom from the cumulative destructive effect of hot spots. We have also found tantalum superior to tungsten for laboratory research work as it is much easier to form into spirals and with properly chosen dimensions is mechanically stiff enough. It seems to have less tendency to lose emission by poisoning than tungsten under our working conditions. Our present spirals are made of 7 inches of 40 mil tantalum wire (5 inches effective for emission) and work at 30 amperes and about 5 volts. No glass is used for insulating the conductors leading current to the filament. This is accomplished with mica pressed between male and female conical sleeves. The filament and its conducting leads (steel tube and axial copper rod) form an assembly which can be slid in the steel shield system which entirely covers it. The smaller sylphon bellows permits this without breaking vacuum by adjusting the nuts on the outermost studs at the extreme cathode end of the tube. Thus the depth of the filament in its shielding box can be adjusted. The $1 \frac{1}{4}$-inch gap between cathode and target can also be adjusted without breaking vacuum by adjusting the nuts on the larger studs which contract or expand the larger of the two cathode sylphons and move the entire cathode assembly axially. A detail of this mechanism appears at the lower right in Fig. 3. The filament assembly with both its electrical connections is entirely insulated from the shield system and the true emission curfent can thus be measured without error from corona or other spurious 'emission which does not hit the target. A biasing voltage can, if desired, also be applied between shield and filament.

\section{$\mathrm{X}$-ray shielding built into tube}

Many pounds of lead have been saved by the incorporation of $\mathrm{x}$-ray shielding into the design of the tube itself. An inch of lead is just as effective near the focal spot as far from it. Removable lead castings in conveniently shaped pieces cover the outside of the steel center section and the exhaust pipe down to the flange for attaching the diffusion pump. X-rays are prevented from escaping into the diffusion pump by a baffle consisting of a central lead disk and an annular lead ring with a short cylindrical flange extending with close fit in the exhaust pipe up to a point well above the lower end of the external lead sheath. The steel drum heads and axially concentric steel tubes each terminated with a well-rounded annular steel plug are autogenously welded in place in the central steel section of the tube and pure lead is cast in the space between drum heads and tubes as shown, the lead being poured into the preheated shell through two small holes which are subsequently plugged and soldered tight. The cones of $\mathrm{x}$-rays which issue through the end openings in these shield tubes are stopped by the lead filled steel disks supported on the target and cathode assemblies, a cross section of one of which appears in the detail at the upper left in front of the gyrating sylphon. We have considered $\frac{3}{4}$ of an inch of lead sufficient shielding for most purposes. Some of the shields which may need to be frequently removed or may for some other reason need more mechanical strength are made of an alloy similar to type metal but richer in lead which we have found almost as satisfactory to machine as brass.

The design of the interior of the tube with the projecting lead filled tubular shields and with the lead filled steel disks on the cathode and target elements accomplishes another purpose beside that of shielding the $\mathrm{x}$-rays in that it 


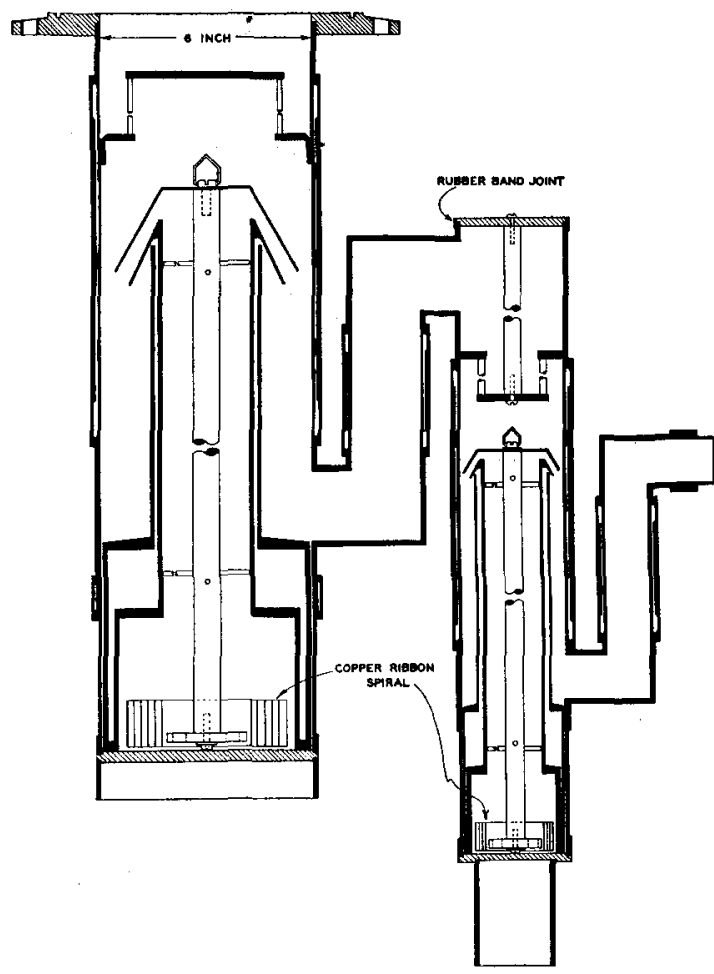

Fig. 4. Cross section of brass Apiezon oil diffusion pump for $x$-ray tube.

concentrates the heavy electrical fields in such a way as to accomplish three other ends. First, the design minimizes the stress transverse to the thickness of the porcelain wall of the tube in regions that could ill withstand such stresses; second, it shields the gasket joints from ion bombardment; and third, it avoids long paths over which charged particles could travel with greater chance of forming ions by collision than over short paths. All sharp convex corners have been rounded off and the steel has been carefully surfaced and highly polished to suppress cold emission.

\section{The windows}

Two short projecting flanged steel tubulations on diametrically opposite sides of the tube are provided for bolting on with a tongue and groove rubber gasket joint any one of several steel disk shaped covers. These covers are provided with several types of $x$-ray and optical windows. One of these disks has a reentrant tube of $3 \frac{1}{2}$ inches inside diameter with an x-ray window $2 \frac{1}{2}$ inches in diameter at its inward end which is thus very close to the target. Another window is rectangular $2 \frac{1}{4} \times 3$ inches of thin aluminum. Still another disk which is generally used on the rear side of the tube has an optical window of Pyrex to afford visual observation of the interior. We contemplate the possibility of adapting our $x$-ray tube to the ring target and ring cathode design used by Kirkpatrick and Ross in which case an aluminum tube would be installed so as to run transversely to the axis of the $x$-ray tube between two steel disk covers bolted in place of the window disks.

\section{The porcelain insulating shells}

These parts were made from standard electrical porcelain bushings of the type known as 75 kilovolt outdoor current transformer bushings. The manufacturers on request furnish these with the metal end rings (for bolt lugs) cemented on so that the porcelain shell projects from $1 / 8$ to $1 / 4$ inch beyond the metal ring. The ends of the porcelain shell are lapped smooth with fine abrasive against a true steel lapping plate to insure a tight fit against the rubber gasket. Unless the porcelain has been mechanically cracked we have had no trouble with these shells leaking. Electrical insulating porcelain has been developed over a period of years so that now an extremely homogeneous nonporous product is the rule. The requirements for a good long lived out-door, electrical, porcelain, insulator happen to be identical to the requirements for a good vacuum leak-proof, electrically insulating, shell for an x-ray tube.

Insulating varnished redwood frames rolling on castors are provided with spring cradles which support the porcelain bushing especially while the tube is disassembled. The spring cradles conveniently support the weight of the bushing while assembling it with its gasket against the steel end flanges and have ample flexibility to permit it to seat itself naturally when, as the tube is pumped out, the atmospheric pressure starts to act. These cradle supports remain in place always.

\section{The $\mathrm{x}$-ray tube diffusion pumps}

Figure 4 shows our diffusion pumps in cross section. These differ but little from other designs now in use with Apiezon "B" oil. The boilers are provided with double walls to reduce heat con- 
duction away from the hot boiling chamber to the nearby regions of the outer pump wall which we found it necessary to maintain cool below the exhaust outlet with a small water jacket, in order to avoid a counter jet of oil vapor opposing the main pumping jet. The restricted thickness of the water jackets encourages turbulent water flow and effective heat transfer. Most of our experience with Apiezon oil parallels closely the findings of Hickman ${ }^{9}$ and we contemplate in the near future modifying the design of our pumps to include his ingenious ideas in which the pumps automatically fractionate their own oil and deliver the light fractions to the higher pressure stages. We have noted the importance of increasing the boiling surface in the liquid phase of the oil and have found that this is easily accomplished by wrapping thin copper ribbon about as wide as the depth of the liquid oil into a flat spiral and placing it so that the ribbon stands on edge in the oil on the bottom of the pump inside the boiler (see Fig. 4). Without this precaution the boiling oil makes a clearly audible clicking noise which we infer means that the oil is "bumping" or boiling in more or less explosive bursts with quiescent periods between the bursts on account of the cooling incident to the sudden explosive loss of latent heat in each "bump." This clearly should make for very ineffective pumping and we have found that a continuously indicating vacuum gauge will invariably indicate serious impairment of vacuum as soon as the heat supply is raised sufficiently to produce the "bumping" noise. With the increased boiling surface and the improved transfer of heat throughout the liquid oil conferred by the use of the spiral of copper ribbon the pumps make a steady frying sound instead of the intermittent bumping.

\section{Charcoal trap and hydrocarbon contamination}

We do not yet feel entirely satisfied with the solution of the problem of hydrocarbon contamination on our target surface. The applications for which this tube is destined make much more rigid requirements in this regard than is the case in almost any other high vacuum "plumbing" equipment we know of with the possible exception of the work of Dr. John

\footnotetext{
${ }^{9}$ K. C. D. Hickman, J. Frank. Inst. 221, 215, 383 (1936).
}

Strong of this institute in coating large astronomical mirrors with aluminum by vaporization. Strong reports far less trouble with hydrocarbon contamination from the Apiezon oil in his pumps than we have experienced. We are unable to say whether the difference comes from the very powerful electron and ion bombardment of all surfaces which takes place in our tube but is absent in his case save in very mild form when he uses a light gas discharge to clean up his surfaces. Certain it is that our visible brown hydrocarbon deposits always appear on bombarded surfaces and especially on the face of the target where they are least desired.

A partial solution to this difficulty is the charcoal trap shown in Fig. 3. The gross oil spray and condensate is prevented from reaching the charcoal trap by two sets of baffle plates each consisting of a disk and an annular ring, the upper, set just below the charcoal trap, being of lead already referred to as an $\mathrm{x}$-ray shield. The charcoal trap which is of the complete obstruction type greatly slows down the pumping speed. The charcoal is retained in the annular space between two concentric cylinders of copper gauze and the gas pumped out must pass through a wall of charcoal grains about three-quarters of an inch thick. For outgassing the charcoal a General Electric "Calrod" heater unit is imbedded in it and electrically connected to a source of power through the wall of the exhaust pipe through leads insulated with mica sleeves and washers and made vacuum tight with "glyptol" lacquer. J. A. Becker and Jay Cox have reported that charcoal, when it absorbs the hydrocarbon products of the pump, transforms them so that they are to a large extent outgassed subsequently with heat as permanent gases readily removed by the pumps. Unfortunately in our case, however, there still remains considerable difficulty from greasy contamination of the tube when the charcoal trap is outgassed, and this type of trap on this account may soon be abandoned for a refrigerant trap supplied with cold brine circulation since Hickman has found that even a moderate lowering of temperature is very helpful. Another resort which we propose to try in the near future is to hold the target at elevated temperature with a heater inserted in place of the cooling water pipe at all times (save 
when the tube is in actual operation) to prevent gradual condensation of the oil on the target.

We have found that a small liquid-air trap placed in the fore vacuum line between the vapor condensation pumps and the mechanical pump is of the greatest value both in increasing the limiting vacuum that the two-stage diffusion pumps will give and for protecting the lubricating oil in the mechanical pump from contamination by volatile vapors slowly distilled from the oil in the diffusion pumps. When the charcoal trap is outgassed after exposure to the atmosphere it gives off water vapor in amazing quantities and we have actually seen this emulsifying the lubricating oil in the mechanical pump so that it resembled mayonnaise, a result which is more effectively prevented by the liquid-air trap than by any other drying agent we have tried.

\section{Knudsen type vacuum gauges}

Two of these gauges are installed, for vacuum measurements on the $x$-ray tube and on the thermionic rectifier valve system, respectively. After much disappointing experience with the McLeod, Pirani and ionization types of gauge the simplicity and reliability of this rugged and inexpensive gauge came as a welcome relief. It has no incandescent filaments to burn out or decompose organic vapors, it requires no expensive electrical meters, has a perfectly stable "zero" or perfect vacuum position which can be checked at any time, indicates pressures as low as $10^{-6} \mathrm{~mm} \mathrm{Hg}$ continuously, measures the pressure of all gases and vapors with the same deflection independent of the molecular weight and involves the use of no fluids like mercury whose vapors must be trapped off and which in the event of an accident may flow into the system and permanently contaminate it. Its construction and advantages have already been fully described by the senior author in collaboration with W. M. Pickels ${ }^{10}$ and are therefore omitted here.

Our Knudsen type gauges will always indicate impairment of the vacuum during operation of the tube with sufficient promptness to permit the operator to shut down the power before a gas discharge occurs in the tube.

${ }^{10}$ DuMond and Pickels, Rev. Sci. Inst. 6, 362 (1935).

\section{Filament current supply and its mercury control} resistance

The thirty-ampere filament current is supplied by storage batteries on top of a redwood insulating stand. The batteries are kept charged during operation by two automobile battery charging generators working electrically in parallel. These are driven with 72 inch rubber $\mathrm{V}$ belts from an induction motor near the floor and at ground potential. The batteries serve to stabilize the voltage of the generators.

In order to control the thirty-ampere filament current a continuously variable rheostat of very low resistance consuming only about one volt is required. This must be capable of fine adjustment as the emission of the filament (and hence the load on the tube) will be extremely sensitive to small variations in this resistance. Paul Kirkpatrick has devised a mercury column rheostat ${ }^{11}$ so satisfactory that its use has become rather general where $\mathrm{x}$-rays are used as a research tool by physicists. This design could not, however, be used in the present case because it occupies considerable space vertically, about twice the depth of the mercury column in fact. In the present case the cathode connections are at a voltage differing from ground in the worst case by 150 kilovolts and the clearance from the ends of such a vertical mercury column to either the floor or ceiling of our laboratory would have been inadequate. We have therefore devised a horizontal type of mercury resistance which may be made either of glass or of steel and which has proven most satisfactory for our purpose. The glass design is made of tubing in the form of a letter U lying with the plane of the letter horizontal. This plane can be tilted by a slow motion worm gear driven by a belt from ground potential the tilt being about a horizontal axis parallel to the straight legs of the U. One of these legs is round in cross section and simply acts as a reservoir of mercury. The other leg has a flattened wedge shaped cross section with the long axis of the wedge vertical. The horizontal mercury column in this tube therefore has a depth which can be varied by adjusting the tilt of the plane of the $U$. The tungsten terminals of this resistance are sealed through the glass

${ }^{11}$ P. Kirkpatrick, J. O. S, A, and R, S, I. 7, 195 (1923). 
at either end of this flattened tube and the resistance is varied not by varying the length of the fluid conductor but by varying its cross section. In the steel design which is now being constructed the heat will be dissipated a little more effectively than in the glass one and the concentration of heat which the glass design exhibits near the tungsten terminals will not occur. The steel rheostat is in the form of a letter $\mathrm{H}$ with the plane of the letter, as before, horizontal. The reservoir stem of the $\mathrm{H}$ is a shelby steel tube of round cross section. The other stem of the $\mathrm{H}$ is steel airplane tubing with a "streamline" cross section (long axis vertical) which we have flattened a little more by compression in a vise. The cross bar of the $\mathrm{H}$ is arc welded into the two stems and it enters the flattened airplane tubing at the very bottom of the trough so that with the proper tilt the mercury will drain out of the trough completely. The flattened airplane tubing has two stainless steel plugs welded into either end and these form the terminals of the rheostat. The minimum and maximum resistance of this rheostat thus correspond to the resistance of the steel airplane tube full of mercury and empty, respectively.

The impossibility of open circuiting this rheostat is an advantage because interruption of the filament current suppresses the emission suddenly and with the load removed the voltage may rise in a surge destructive to other apparatus.

\section{Surge protection for electrical measuring instru- ments}

The high power available from our high tension direct current power supply system is sufficient to furnish about two kilowatt-seconds on short circuit simply by the discharge of the stored energy in the last stage of condensers of the electrical filter. The limiting currents are so high in the case of an accidental gas burst in the $\mathrm{x}$-ray tube, which may frequently occur while the tube is being outgassed, that protection for the milliammeter is indispensable. Fig. 5 shows how we connect a small neon lamp N.L. in conjunction with a reactance, $L$, a fuse, $F$, and a breakdown film arrestor, $F . A$., to protect the instrument, $M . A$. For most ordinary surges the neon lamp effectively protects the instrument without the nuisance of having to interrupt the

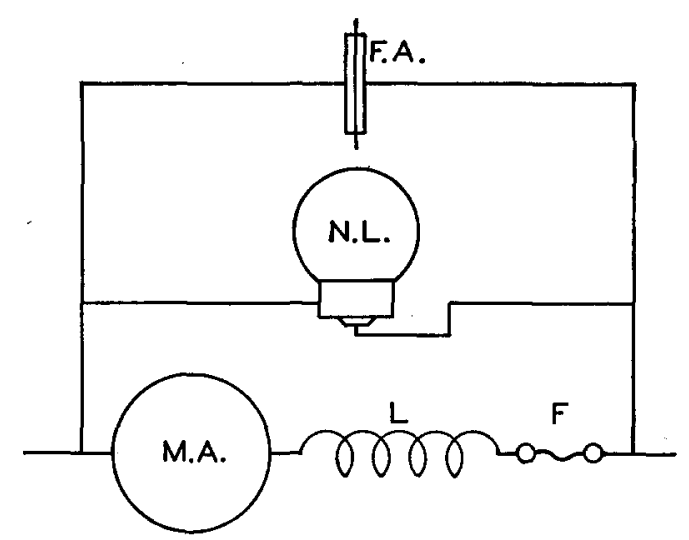

FIG. 5. Meter protective precautions.

voltage and replace a fuse. The film arrestor and fuse protect the instrument against rarer and more violent accidental discharges. The neon lamp also provides an excellent visible signal even when surges occur which are to small to appreciably flick the milliammeter needle.

\section{Water-cooling supply for target}

Our present supply of cooling water for the target comes from a large metal basin on the roof of the building. The system consisting of this basin and the 1-inch copper pipe which conducts the water down a height of three stories outside the building to an insulating entry point through a window is supported entirely on porcelain outdoor compression type insulating legs. The supply of water falls into the basin on the roof from a shower bath spray nozzle and this finely divided falling column of water is a good insulator so that the potential of 150 kilovolts at which the cooling water system is maintained gives rise to a negligible current to ground. The hot water discharged from the target likewise falls from an outdoor nozzle freely into a grounded basin. The head furnished by the three stories from the basin on the roof to the lower discharge nozzle at present gives us 2 gal. per minute.

Inlet and outlet rubber hoses connect directly to the gyrating target stem with ordinary hose couplings. These hoses are firmly held in a large clamp supported in a projecting frame of angle iron clearly visible at the extreme left-hand end of the tube in the general view of the laboratory room, Fig. 6. The clamp prevents vibrations caused by the gyration from being transmitted outside of the tube structure. 


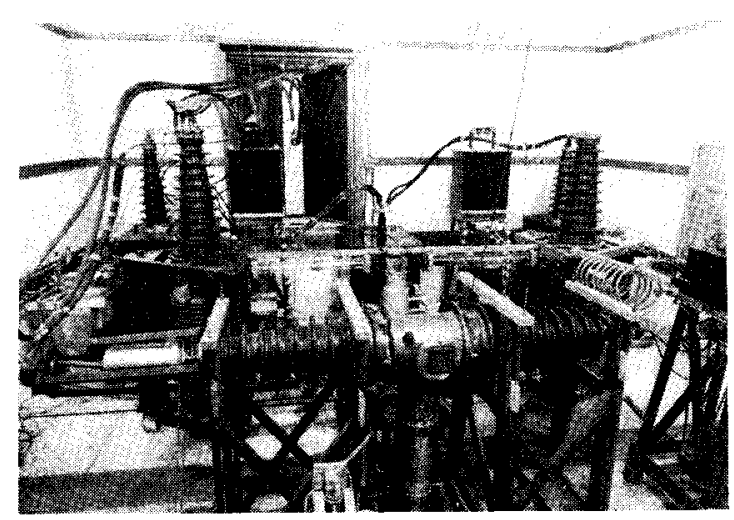

Fig. 6. General view of Watters Memorial Research Laboratory with $30 \mathrm{kw}$ high intensity gyrating target $\mathrm{x}$-ray tube and its high tension d.c. power supply equipment.

\section{Gaskets}

Rubber gasket joints have been used almost exclusively throughout the vacuum design. When such gaskets are merely compressed between flat surfaces the secret of success we find is in cutting the gasket so that its radial width is thin and very uniform. In a gasket of irregular width thin spots are shielded from receiving sufficient compression by the wider and less yielding adjacent portions so that leaks or even "blow ins" will occur in the thinner regions. The gasket rubber should be secured to a board with many closely spaced thumb tacks both inside and outside the region to be cut. It is then cut by hand with a sharp wet knife on a radius bar. A rubber gasket supported in a well fitted tongue and groove joint is extremely satisfactory and is far less exacting as to cutting. For fore vacuum lines a properly wrapped bandage joint ${ }^{12}$ can be made with electricians' gum rubber

${ }^{12}$ We owe this technique to C. C. Lauritsen and $R$. Crane. The beginning end of the gum tape is first thinned by stretching. It is then wrapped straight around the pipes a little more than one whole turn so that it laps about equally over both sides of the fissure. From here on it is wrapped so that it overlaps on itself about half its width for a distance of about an inch along the pipe first to right (say) then all the way back over the wrapping to a point one inch to the left of the fissure then back to the middle where it is cut off and the end pressed tightly against the underlying layers to give good adherence. If this has been carefully done there are in the joint now only two critical places the "cross overs" one at either end of the wrapping where the direction of spiraling was reversed so that the tape crossed back over itself. At these points where the tape climbs up over its own thickness on the turn below a small duct spiraling in along the edge of the tape may lead all the way to the fissure to be bandaged. These cross over points should be thoroughly squeezed tape for joining two abutting pipes so that with a little coating of shellac it will never leak. A plain rubber band (say $\frac{1}{2}$ inch wide) snapped over the fissure between the flush abutting ends of two tubes and then coated with shellac will always make a tight joint. This latter is not as strong mechanically, however, as the bandage if slight relative motion of the pipes may be expected. It is probable that lead fuse wire gaskets could have been used in place of rubber but some unfortunate early breakages of expensive porcelain in an effort to make such fuse wire joints tight have perhaps unduly prejudiced the present authors against them. It is essential, of course, to protect the rubber gaskets both from heat and from ion bombardment to avoid outgassing as well as to prevent them from being destroyed.

Large gasket joints are if anything easier to

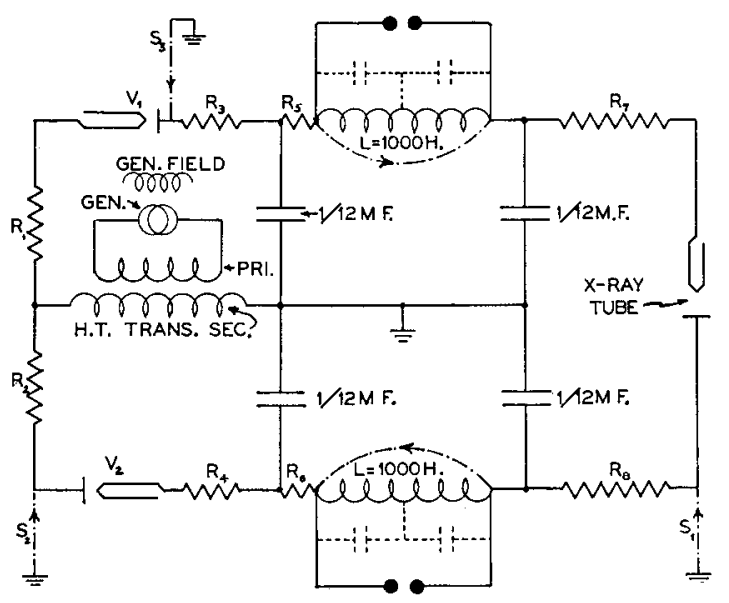

FIG. 7. Circuit diagram of power supply system for 30 kw x-ray tube equipment. The zigzag lines represent the damping resistances consisting of short segments of rubber garden hose containing flowing tap water. These stabilize the circuit against heavy surges of current. $S_{1}$ is the large water supply for the $\mathrm{x}$-ray tube target. It also supplies water for the resistances. $R_{8}, R_{6}$ and $R_{4}$, the dot and dash arc representing about twenty feet of hose electrically shunting the inductance in order to conduct water to $R_{6}$ and $R_{4}$. Similarly $S_{3}$ is a water supply through a long 200 foot helix of hose for cooling the plate of the valve tube $V_{1}$ and to supply water to $R_{3}, R_{5}$ and $R_{7} . S_{2}$ is a water supply helix for the plate of $V_{2}$ and for resistances $R_{1}$ and $R_{2}$. The dotted condensers shunting the chokes are to resonate with the two principle ripple frequencies but are not yet installed.

with the thumb nail to close up the duct and should be thoroughly shellacked and carefully observed in case the shellac may "suck in" before it has dried. Needless to say it is wise to make the cross over points at a convenient location for observation and painting. 


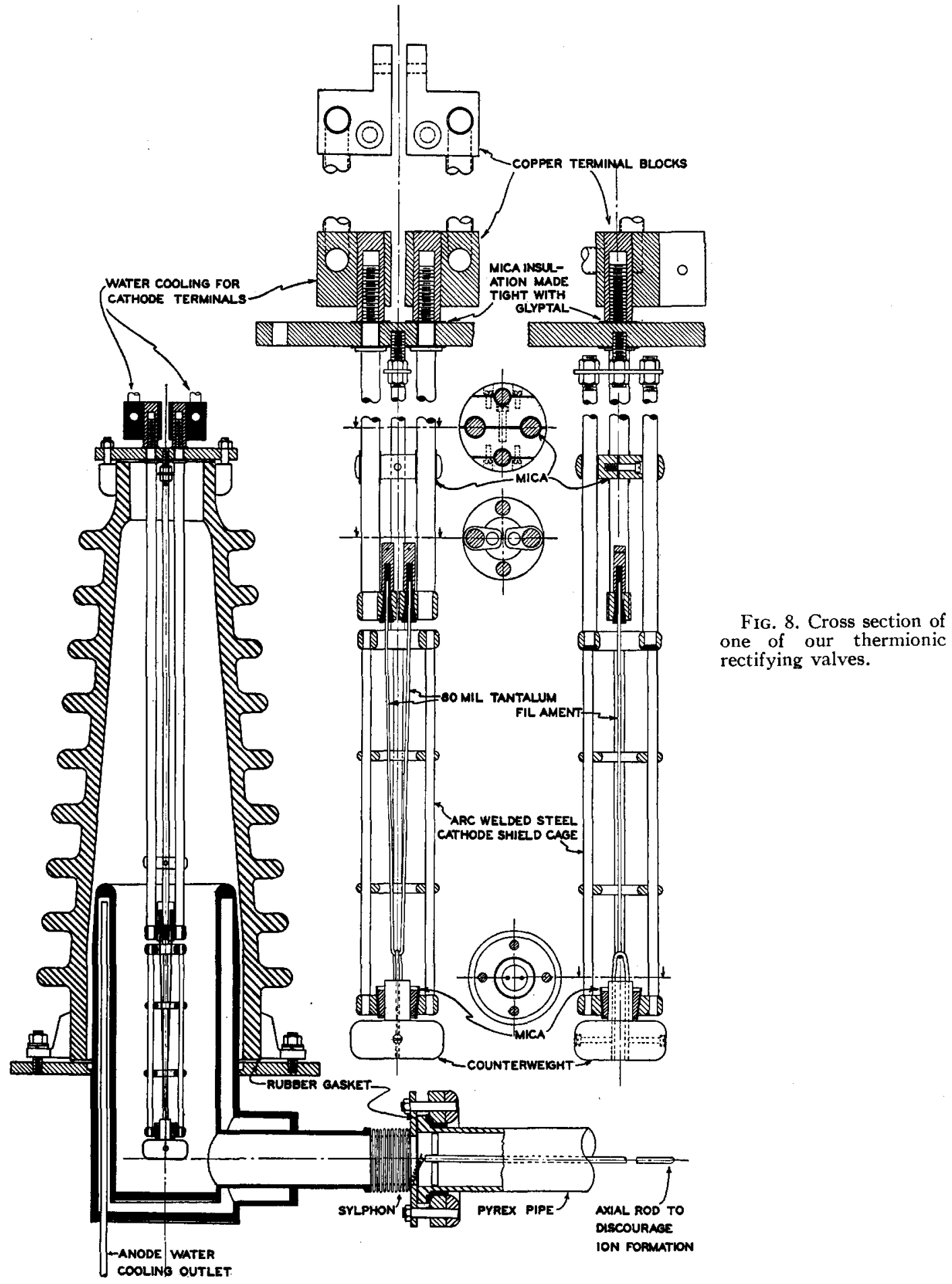

make tight than small ones. The compressive force from atmospheric pressure tending to make the gasket tight increases as the square of the diameter of the gasket while the length of the gasket only increases as the first power as does also the chance of a leak. 


\section{The High Tension p.c. Power SUPPLY}

\section{The circuit diagram}

Figure 7 is a diagram of the two wave rectifying circuit we employ to supply rectified and filtered high voltage d.c. to the tube. The diagram with its caption are self explanatory.

\section{The thermionic valves}

Figure 8 is a cross section through one of the rectifying valves. The anode shell or plate is a double-walled steel cylinder supplied with cooling water. This cylinder projects well up into the interior of the porcelain insulator (a porcelain bushing exactly like the ones used on the x-ray tube). The water cooling is necessary to dissipate the heat of more than a kilowatt radiated from the thermionic filament. The heat generated by the electron emission currents of from one to two amperes bombarding the plate is, as it should be, relatively small. This is because under correct operating conditions the instantaneous current demanded of the valve tube filament emission by the $\mathrm{x}$-ray tube load and the filter condensers is kept considerably lower than the saturation value so that the P.D. from filament to plate in the thermionic valve during the electrically conducting interval is low.

Our design very effectively protects the large rubber gasket at the bottom of the porcelain bushing both from heat and ion bombardment. The steel parts are arc welded together and the surfaces in the vacuum are carefully finished and polished to avoid cold emission. With 150 pounds per square inch of air pressure in the water jackets the job was carefully tested for leaks in the welds after the surfaces had been finished by painting the surface with soap suds.

The cathode design for the thermionic valves has undergone many modifications before the present permanently satisfactory design was obtained. As in the $\mathrm{x}$-ray tube filament this experience has taught us the value of short, thick, rugged, high current low voltage cathode design. The present cathodes are of $80 \mathrm{mil}$ diameter tantalum wire in the shape of an elongated $U$. The heating current is 100 amperes and the power input a little over one kilowatt. The upper ends of the tantalum are clamped into the heavy copper blocks which terminate the half inch diameter copper conductors with a small steel clamp exactly resembling a collet chuck on a lathe. This design is excellent for the heavy currents involved both thermally and electrically and we have never yet had one of these tantalum filaments weld tight in its collet. The heat from the filament conducted up the copper current conductors makes necessary a little circulating water cooling at the top to protect rubber gaskets and the mica insulated current lead-in joints (made tight with glyptol lacquer). The heavy filament heating currents are supplied from especially designed oil immersed filament transformers built by ourselves capable of insulating this current supply from ground against 150 kilovolts. The insulating terminal bushings of these transformers can be seen in the two far corners of the room in Fig. 6.

The U-shaped cathode filament is shown surrounded with a four barred grid which serves a four fold purpose. First this grid shields the filament from heavy electric fields which might distort it. Second we believe that it materially protects the filament from destructive ion bombardment during outgassing as the arc probably strikes more readily to the grid than to the filament. Third it serves as a support and guide for the counterweight hanging on the bottom of the filament to prevent this from swinging. (This counterweight allows the filament to expand and contract while maintaining a slight tension on it.) Finally the rings at uniformly spaced intervals on this grid baffle a great deal of the heat radiation from the filament which would otherwise go obliquely upward through the open end of the steel anode plate and overheat the inner walls of the porcelain bushing. The voltage required to saturate the filament emission is only moderately increased by this grid.

\section{Initial outgassing of thermionic valves}

Efforts to outgas the thermionic valves under their normal high inverse voltage operating conditions are very tedious and liable to be destructive to the filament. A simple but important experiment which it cost us a great deal of time to discover is to outgas the valves before applying normal operating voltage to them by 
applying about 5000 volts a.c. from a $10 \mathrm{kw}$ transformer direct from filament to plate so that with the full filament emission current from five to ten kilowatts are dissipated on the plate. The water cooling is maintained on the plate during this process. This thoroughly bakes out all the interior surfaces of the valve so that when the high voltage is applied no outgassing occurs and no destructive arcs are formed. A quick acting circuit breaker opens the primary current supplied to the 5000 volt transformer in this outgassing process the instant an arc starts to form and before the ion current has reached serious proportions by means of a relay in the transformer secondary set for two amperes. This method of outgassing is very harmless in its effects on the filament which shows no subsequent damage or pitting. It affords an enormous and very welcome saving in time for the high voltage can be applied immediately after this treatment.

\section{Insulating glass exhaust pipes for thermionic valves}

With the circuit of Fig. 7 both terminals of each thermionic valve are at potentials very different from ground potential during various stages of the cycle. As it is convenient to have the vacuum pumping system for the valves at ground potential we have provided electrically insulating exhaust pipes 3 inches in internal diameter and three feet long of Pyrex glass (visible in Fig. 6) to connect a single two stage Apiezon oil pumping system with both thermionic valves. These glass pipes, provided with end flanges for clamping them against gaskets, are made by the Corning Glass Company as acid pipes for use in industrial chemistry.

With the electrical field from the P.D. of 150 kilovolts extending over the meter length of these pipes very destructive electrical gas discharges occurred in them. The long paths which these pipes afforded for ions strongly encouraged ionization by collision unless the vacuum was maintained at very long mean free paths. This trouble we cured easily by installing two quarterinch aluminum rods extending along the central axis of the pipe the one connected to the thermionic valve anode the other to the grounded pumps. The gap near the middle of the glass pipe between the hemispherically rounded ends of these aluminum rods is only about four inches long and the field from the 150 kilovolts is entirely in the region of this gap. A vacuum many times poorer can be tolerated without visible gas discharge on account of this shortened gap, an effect always astonishing to those unfamiliar with discharges in high vacuum.

\section{Cooling water for valve anodes}

This is supplied at a sufficient rate to wash away the relatively small amount of heat radiated to the anode from the valve tube filaments, one kilowatt, through a double helix consisting of two hundred feet of $\frac{1}{4}$ inch inside diameter and $\frac{5}{8}$ inch outside diameter hose. One hundred feet of this conducts the cool water from ground potential to the anode. The other hundred feet takes the warm water back to the drain. This helix can be seen in the photograph of Fig. 6 underneath the thermionic valves between the three porcelain supporting legs which carry the valve structure. The loss of current down this helix is from six to ten milliamperes depending on the conductivity of the tap water.

\section{The electrical filter}

The two half-waves from the transformer that have been rectified to a pulsating voltage by the thermionic valves maintain the first stage of condensers charged to a nearly constant voltage which at full load is afflicted with a ripple of about 6000 volts total amplitude. The steady load current of 100 milliamperes for the x-ray tube together with the alternating charging current demanded by the second stage of condensers passes from the first stage condenser through a pair of 1000 henry oil immersed choke coils in which the reactive effect of the alternating component of the current neutralizes all but one percent of the first stage voltage ripple.

The contemplated addition of small condensers shunted across the chokes so as to form resonant circuits for the ripple frequencies should, according to our experience with smaller power supply units, greatly improve the ripple suppression. Of course, at more moderate loads than 100 milliamperes, the ripple amplitude is proportionally reduced. On account of the 100 milliampere d.c. component in the current through the chokes we have provided their nearly closed cores with an air gap of about 2.5 millimeters. 
In computing the choke design we have followed the very ingenious paper of $\mathrm{Hanna}^{13}$ for the design of filter chokes to carry a.c. and d.c. current simultaneously. The chokes with their protective sphere gaps on top are clearly visible in the general view of Fig. 6 in the extreme background as black boxes on high insulating legs.

The condensers consist of twelve cylindrical Dubilier units each with a rating of $\frac{1}{4} \mathrm{mf}$ and $50 \mathrm{kv}$. These are stacked in four columns of three units each. Each of these stacks good for $150 \mathrm{kv}$ with a capacity of $1 / 12$ microfarad is represented in the diagram of Fig. 7 by a single condenser symbol. When a substantially continuous voltage is applied to condensers in series the distribution of voltage between them is determined by their leakage conductances and the danger is therefore always present that one of the three condensers in our stacks may be subjected to more than its share of voltage stress. Scarcely anything is more irreparable than a punctured condenser and so tó insure against such calamities we have provided sphere gaps (made of smooth copper plumber's float spheres) which shunt every condenser unit and are set only very slightly wider than the breakdown distance for $50 \mathrm{kv}$. These are connected to the condenser stacks through carbon resistance rods to limit the flash-over current. The condenser stacks are insulated from the floor on which they stand so that the a.c. current which flows through the condensers to ground can be measured in order to estimate the magnitude of the ripple or study its form with an oscillograph. This also permits the application of an idea of P. A. Ross who has shown in unpublished work that a ripple can be largely neutralized by applying one or more a.c. voltages of the correct intensity, phase and frequency in series between the filter condensers and ground.

\section{Transient or surge suppression}

Referring to the diagram of Fig. 7 it can easily be seen that if either or both thermionic valves become electrically conductive in the inverse sense (either because of evolution of gas or by cold emission) or if the $\mathrm{x}$-ray tube suddenly permits a surge of current from a similar cause, then the stored energy in the condensers will

\footnotetext{
${ }^{13}$ C. R. Hanna, J. Am. I. E. E. 46, 128 (1927).
}

furnish a very destructive surge of current which may rise for a brief period to exaggerated intensities. The inductive effects from such surges in neighboring circuits and conductors can be surprisingly large frequently destroying instruments and breaking down insulation. Such effects which present no problem in equipment of smaller power rating are very serious in the present instance. Under ordinary circumstances our set operates very quietly indeed but a single exceptional case (which generally occurs while outgassing) may be very expensive and destructive. We have met this difficulty by introducing some resistance (represented in Fig. 7 by zigzag lines) in every connecting link in the high voltage circuit. A brief analysis will show that every one of these is required for surge suppression in the event of one or another type of failure. The resistances which in normal operation must carry the heavy momentary currents supplied by the valve tubes to charge the condensers every half cycle are necessarily of lower value than those in parts of the circuit that carry only the 100 milliamperes load current to the $x$-ray tube. All of these resistances consist of columns of tap water flowing in rubber hose. Some of this water comes to parts of the circuit at such potential that it can be supplied through the helices which also supply cooling water to the thermionic valve anodes; some of it is part of the same water supplied to the $\mathrm{x}$-ray tube target. These hose resistances can be varied at will because they have been made up in short segments connected with a number of permanent metallic connections. As many segments of hose as desired can

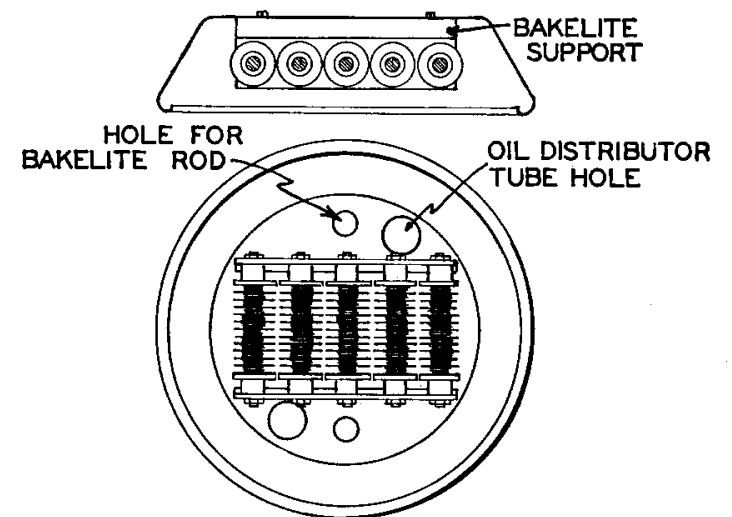

FIG. 9. One of the modified aluminum pie tin units with its five spools of one megohm each (metallic wire wound resistance). 


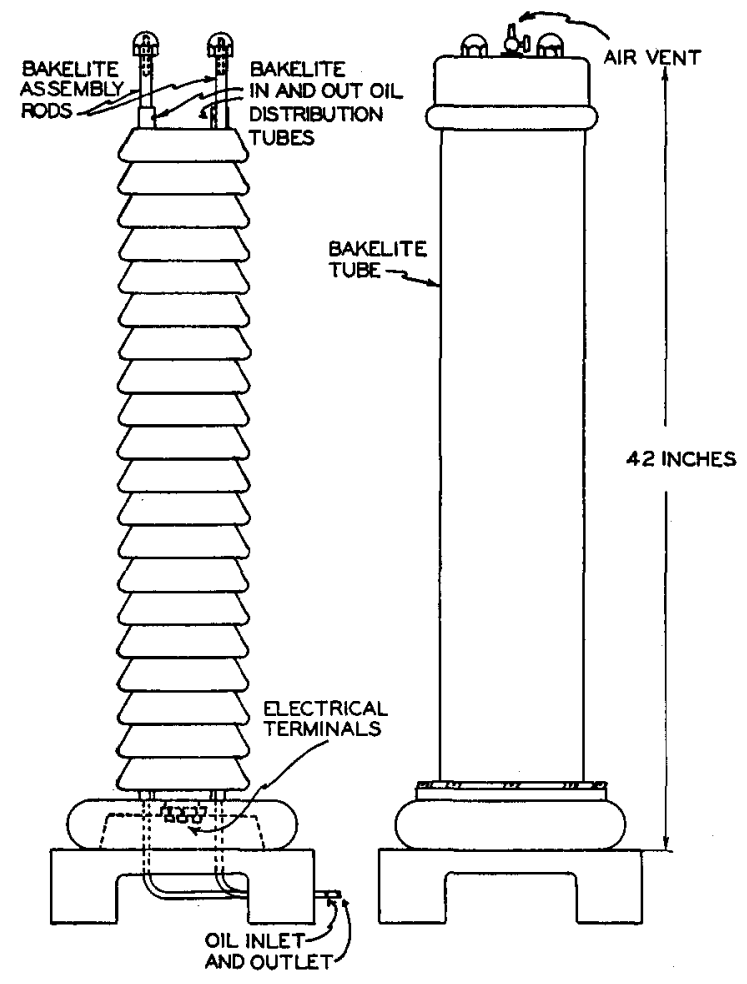

FIG. 10. High tension resistance towers. The one on the left has the Bakelite shell removed to show the pie tin units the uppermost one of which has also been removed from the Bakelite rods.

thus be short circuited electrically and at some points only a few inches length of hose are in actual use as a resistance a quite sufficient amount to furnish the requisite damping. Our tap water has a resistivity such that a $3 / 4$ inch hose has about $60,000 \mathrm{ohms}$ per foot and since it is nearly always necessary to have two hoses electrically in parallel (since the water must come and go) this means about 30,000 ohms per foot of the double conductor. These hoses can be clearly seen in the general view, Fig. 6.

\section{The primary power supply set}

Our primary source of power is an alternating current generator coupled to a 60 horsepower synchronous driving motor. Each machine has its own directly coupled d.c. exciter to supply its field current. The use of this set very effectively protects our power supply from fluctuations in voltage caused by local fluctuations in load on the city mains. Since the alternator has ten poles while the synchronous motor has four we have the extra advantage of the higher frequency of
150 cycles per second which makes the problem of ripple filtering much easier. A third advantage gained by this motor generator set is the flexibility it affords as to control of the voltage supplied to the high tension transformer by remote rheostat control of the field current of the alternator.

The voltage output by this alternator exhibits small fluctuations of a very random nature of the order of one part in a thousand which we believe come from tiny fluctuations in the power supply frequency. This effect varies greatly on different days being almost completely absent at times. A vacuum tube robot control now under construction should effectively suppress this.

\section{Voltage Measurement}

The projected work on the precision determinations of the "short wave-length limit of the continuous $x$-ray spectrum requires the use of a high resistance to make precision measurements of the high voltage applied to the $x$-ray tube. We have therefore decided to build this resistance so that it can be used generally as a voltmeter for the less precise measurements also.

Referring to Figs. 9 and 10 the resistance consisting of 200 metallic wire wound units of one megohm each (manufactured by the Shallcross Company) is mounted in small saucepan shaped aluminum corona shields with five megohms in each pan. The pans were made at very low cost out of deep aluminum pie pans whose shape we modified by spinning near the lip so that instead of flaring outward they curve inward with a large smooth radius of curvature. These pans are provided with appropriate holes so that they can be stacked one above another like a pagoda on a pair of parallel vertical Bakelite rods with short Bakelite tubes to space them apart. The entire stack of pans is contained in a tall Bakelite tube fitting into gaskets in grooves in the corona shields of metal which form the top and bottom of the container. Transformer oil is circulated through this container by means of a pair of vertical Bakelite pipes piercing through the entire stack of pans and provided with small side outlet and inlet holes so as to furnish an entry and an exit for oil to each pan. There are two such stacks of resistance units with 100 megohms in each. Each stack has 21 pans the 
lowest pan containing not megohm units but several spools of smaller resistance across which an accurately known fraction of the total voltage applied to the stack can be measured with a potentiometer. The oil is circulated by a small motor driven gear pump and the heat removed from the resistance stack is transferred from the oil to running water in a heat exchanger. This continual circulation and cooling of the oil insures constant temperature throughout the entire height of each stack. Intermediate resistance taps of $5,10,10,25$, and 50 megohms are provided along the length of each stack so that a wide variety of total resistances can be obtained to suit the voltages to be measured in the case of precision measurements. Each stack measures the voltage from one end of the x-ray tube to ground. Rough values of this voltage for ordinary purposes can be read directly and continuously by reading the current flowing down the entire stacks to ground with a meter. For precision work however the combined P.D. across the two low resistances at the foot of each stack is to be measured with a potentiometer connected as indicated in Fig. 11. In order that this will give the high voltage applied to the tube even in the presence of unbalanced voltage on the two stacks it is evident that the resistance ratios of all corresponding taps in the two stacks must be as accurately as possible identical. ${ }^{14}$ Only these ratios need be determined accurately.

The lowest pan in each stack beside containing resistance taps for potentiometer measurement also contains a tap furnishing a much higher voltage of order 500 volts depending for its exact value on the high voltage maintained on the $x$-ray tube. This voltage is to be balanced against a very steady d.c. source of equal value and the difference, which will measure the fluctuations in the actual high voltage applied to the $\mathrm{x}$-ray tube will be used as a "signal" to actuate the grid of the first stage of amplifier tubes of the voltage regulating robot mentioned before. This robot is to control the voltage of the alternating current generator by applying to its armature an appropriately varying small extra load. This

${ }_{14}$ In a recent paper DuMond and Bollman (Phys. Rev. 51,400 (1937)) have outlined practical methods they have used successfully for calibrating such resistance ratios in the case of a pair of 14 megohm stacks.

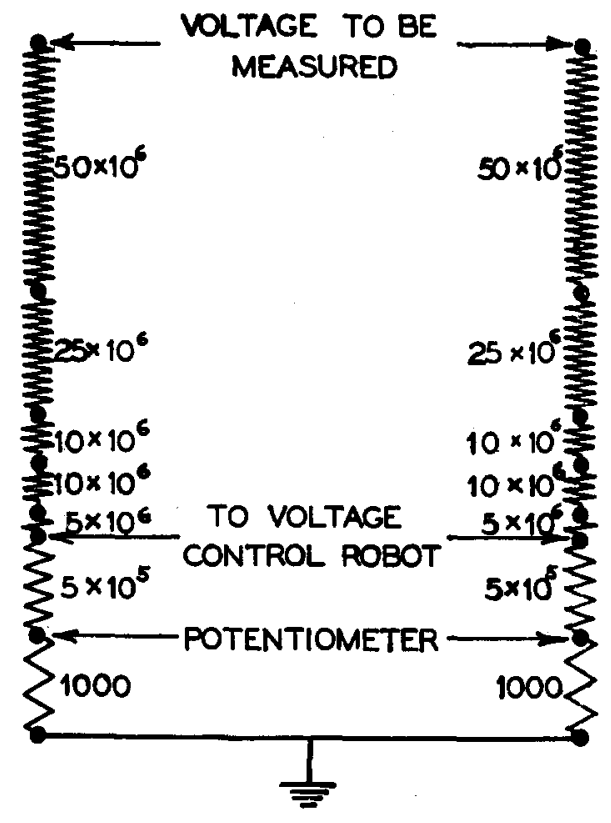

FIG. 11. Diagram of connections to high voltage resistances for precision measurements of the high voltage.

method is preferred to a regulation by means of the alternator field which might prove too sluggish.

\section{Acknowledgments}

Nearly all the parts of the equipment here described were built in our own shops over a period of several years by the writers and their co-workers many of whom have since left this institute. Among those whom we wish especially to thank for their long continued, devoted and able assistance are Mr. Eric Howse, Mr. Bernard Locher, Mr. Lowell Hand, Mr. William Pickels and Dr. Vernon Bollman. We are grateful also for the briefer but equally generous aid given by Mr. William Harper and Mr. Maxwell Kelch.

The equipment we have described is known as the Watters Memorial Laboratory equipment of California Institute of Technology. The funds which made the construction of this equipment possible and which have for several years also supported a number of pieces of experimental research in pure physics were given to this Institute by Dr. Leon L. Watters of New York City as a memorial to his wife Frances $\mathrm{H}$. Watters and it is a pleasure to here express our gratitude for his generous gift. 